\title{
Nasal Carriage Rate of Methicillin Resistant Staphylococcus aureus (MRSA) among Civil Hospital Health care workers
}

\author{
Vaidya Rutvi ${ }^{1}$, Patel Sangeeta. $D^{2 *}$, Bhatt Sima. $K^{3}$, Patel Piyush. $A^{4}$
}

\section{Vaidya Rutvi ${ }^{1}$, Patel Sangeeta. $\mathrm{D}^{2 *}$, Bhatt Sima. $\mathrm{K}^{3}$, Patel Piyush. $\mathrm{A}^{4}$}

\section{'MBBS student, GMERS Medical} College, Gandhinagar, Gujarat, INDIA. 2Department of Microbiology, GMERS Medical College,Gandhinagar, Gujarat, INDIA.

${ }^{3}$ Department of Microbiology, GMERS Medical College, Gandhinagar, Gujarat, INDIA.

${ }^{4}$ Department of Pathology, GMERS Medical College,Sola, Gujarat, INDIA.

\section{Correspondence}

Dr. Sangeeta D. Patel,

13, Mandakini Society, K. K. Nagar Road, Ghatlodiya, Ahmedabad-380061, Gujarat

Ph.no: 9428350579

Email: sanginfriend@yahoo.co.in

\section{History}

- Submission Date: 25-03-2016;

- Review completed: 14-08-2016;

- Accepted Date: 30-09-2016.

DOI : 10.5530/ijmedph.2016.4.7

\section{Article Available online}

http://www.ijmedph.org/v6/i4

\section{Copyright}

(C) 2016 Phcog.Net. This is an openaccess article distributed under the terms of the Creative Commons Attribution 4.0 International license.

\begin{abstract}
Context: Staphylococcus aureus is a common cause of community and hospital acquired infections. One of the important sources of nosocomial infection is staphylococcus, present in nasal carriage among hospital personnel. Emergence of drug resistant strains especially methicillin resistant Staphylococcus aureus (MRSA) is a serious problem in hospital environments. Aims: To isolate Staphylococcus aureus from the nasal swabs of healthcare workers (HCWs) and to study their antimicrobial susceptibility including methicillin resistance pattern. Settings and Design: A hospital based prospective study. Methods and Material: A total of $150 \mathrm{HCWs}$ who were involved in the management of critically ill patients from the intensive care unit, Operation Theatre (OT) and postoperative wards were screened for MRSA by collecting their nasal swabs. All the samples were collected by trained second year MBBS student of the same institute. S. aureus was identified by standard methods. Antibiotic susceptibility test was performed by modified Kirby-Bauer disc diffusion method. MRSA was identified by using a cefoxitin $30 \mathrm{mcg}$ disc and it was interpreted according to the CLSI guidelines. Statistical analysis used: The findings were statistically analyzed by using the Chi-square test. Results: Out of 150 healthcare workers, $33(22 \%)$ were nasal carriers of S. aureus and among them 9 (27.2\%) were carrier of MRSA. Overall nasal carriage rate of MRSA was $6 \%$ (9/150). Highest MRSA carriage rate of $6.5 \%$ (3/46) was found among doctors. The antibiogram of the MRSA isolates revealed that resistance to Amoxiclav (100\%), Erythromycin (45\%) and Gentamycin (40\%). All the MRSA isolates were sensitive to Linezolid $(100 \%)$ and vancomycin (100\%). Conclusions: Screening and decolonization may be effective in decreasing the MRSA carriage rate among the HCWs. Standard infection control precautions should be employed in the professional practice to minimize either the carriage or the transmission rate.
\end{abstract}

Key words: Health care workers, Nasal swab, Nasal Carriage, Staphylococcus aureus, MRSA, Antibiotic Resistance.

\section{INTRODUCTION}

Staphylococcus aureus is a common cause of community and hospital acquired Infections. One of the important sources of staphylococci for nosocomial infection is nasal carriage among hospital personnel. ${ }^{1}$ Methicillinresistant S. aureus (MRSA) is currently the most commonly identified antibiotic resistant pathogen in the world. Treatment of infection caused by S. aureus has become most difficult since the occurrence of methicillin resistance, as MRSA strains are resistant to all $\beta$-lactam antibiotics thereby significantly limiting the treatment options. ${ }^{2-5}$ With a few exceptions, the incidence of nosocomial infection caused by MRSA continues to increase worldwide. ${ }^{6}$ Infections caused by MRSA strains are associated with longer hospital stay, prolonged antibiotic administration and Higher cost than infections caused by methicillin-susceptible Staphylococcus aureus. ${ }^{7}$ Colonized patients, colonized healthcare workers (HCWs) and contaminated environmental surfaces can act as a reservoir for the spread of MRSA to the other patients and other
HCWs. ${ }^{8}$ Identification of patients and healthcare workers colonized with MRSA, combined with hand hygiene and other precautions have been shown to be effective in reducing the transmission and control the spread of MRSA. ${ }^{9}$

Subjects and Methods: This study was carried out during six month period from April 2014 to September 2014

Inclusion criteria: In this monocentre study, all the HCWs who were involved in the management of critically ill patients of the intensive care unit, postoperative surgical wards, major Operation Theatre (OT) of the civil hospital, Gandhinagar ,Gujarat, India were selected for the study after obtaining their informed consent. These all HCWs are working only in civil hospital, gandhinagar, Gujarat, India.

This study was approved by the ethical review committee of the hospital.

Exclusion criteria: HCWs with history of upper respiratory tract infection, recent nasal surgery, use
Cite this article: Rutvi V, Sangeeta PD, Sima BK, Piyush PA. Nasal Carriage Rate of Methicillin Resistant Staphylococcus aureus (MRSA) among Civil Hospital Health care workers. Int J Med. Public Health. 2016;6(4):180-3. 
of nasal medication, or antibiotic therapy were excluded.

Age, sex, work category, duration of working in the critical care unit, prior hospitalization, current skin infection/other illness and other relevant information about the participants were obtained in a proforma which was specifically designed for this purpose.

\section{Nasal swab collection}

A sterile cotton swab with sterile test tube was used for sample collection. The sample was collected by rotating the Sterile cotton swabs gently for five times on both nares of the study participants and transported in a sterile test tube to the laboratory.

\section{Culture and identification}

Swabs inoculated on to Nutrient agar and Mannitol Salt Agar (MSA) within one hour of collection and incubated at $37^{\circ} \mathrm{C}$ for $24 \mathrm{hrs}$. Catalase and Coagulase test were performed from the isolated colonies in the nutrient agar plate. These are the only available techniques for identification of Staphylococcus aureus in our institute. Catalase and Coagulase test positive and mannitol salt fermenting colonies were diagnosed as S. aureus. ${ }^{10}$

\section{Antimicrobial susceptibility testing}

All the isolated Staphylococcus aureus strains were tested against different antimicrobial agents by the modified Kirby Bauer disc diffusion method. Commercially available antibiotic discs were used, which were obtained from HiMedia Laboratories Ltd. The antimicrobial penicillin (10U), amoxyclav (20/10 mcg), erythromycin (15mcg), gentamicin (10mcg), amikacin (30mcg), ciprofloxacin (5mcg), cotrimoxazole $(1.25 / 23.75 \mathrm{mcg})$, vancomycin $(30 \mathrm{mcg})$, linezolid $(30 \mathrm{mcg})$ and tetracycline $(30 \mathrm{mcg})$ were used. The zone diameters were interpreted according to the Clinical Laboratory Standards Institute (CLSI) guidelines. Staphylococcus aureus were further screened for Methicillin resistance by the Kirby-Bauer method by using cefoxitin $(30 \mathrm{mcg})$ discs according to the CLSI guidelines ${ }^{11}$.If the inhibitory zone diameter $\leq 21 \mathrm{~mm}$ around the cefoxitin disc, the strain of staphylococci was interpreted as MRSA strain.

The findings were statistically analyzed using descriptive statistics, Chi-square test (X2) and p-value ( $<<0.05$, statistical significant).

Results: In our study 150 HCWs were screened for MRSA, among them $92(61.3 \%)$ were females and $58(38.6 \%)$ were males. (Table 1$)$

Out of $150 \mathrm{HCWs}, 65(43.3 \%)$ were nurses, 46(30.6\%) were doctors and $39(26 \%)$ were housekeeping staff. They all were screened for nasal MRSA carrier.

From the 150 nasal swab samples, in 33 (22\%) Staphylococcus aureus were isolated. Among 33 Staphylococcus aureus, Methicillin resistant Staphylococcus aureus (MRSA) were 9 (27.2\%).

Out of 150 healthcare workers, 33(22\%) were nasal carriers of S. aureus and among them $9(27.2 \%)$ were carrier of MRSA. Overall nasal carriage rate of MRSA was $6 \%(9 / 150)$. Highest MRSA carriage rate of $6.5 \%$ (2/26) was found among Doctors. (Table 2)

Based on their areas of work, the highest rate of s. aureus carriers were found in HCWs of intensive care unit (ICU) $(34.61 \%, 9 / 26)$ and MRSA carriers were found in HCWs of emergency department $(15.3 \%, 2 / 13)$ (Table 3).

Antibiotic susceptibility test were done for 33 isolates of Staphylococcus aureus. 12 antibiotic discs were used. Their susceptibility patterns were shown in Table 4.

The antibiogram of the MRSA isolates revealed that resistance to Amoxiclav (100\%), erythromycin (45\%) and Gentamycin (40\%). All the
Table 1: Healthcare workers screened for Methicillin Resistant Staphylococcus aureus

\begin{tabular}{cccc}
\hline Category & Frequency (\%) & Male (\%) & Female (\%) \\
\hline Doctors & $46(30.66 \%)$ & $28(18.66 \%)$ & $18(12 \%)$ \\
Staff Nurses & $65(43.33 \%)$ & $21(14 \%)$ & $44(29.33 \%)$ \\
House Keeping & $39(26 \%)$ & $09(6 \%)$ & $30(20 \%)$ \\
Total & 150 & 58 & 92 \\
\hline
\end{tabular}

Table 2: Distribution of Staphylococcus aureus and Methicillin Resistant Staphylococcus aureus Carriers among Healthcare Workers

\begin{tabular}{cccc}
\hline Category & $\begin{array}{c}\text { No. sampled } \\
(\text { no }=150)\end{array}$ & S.Aureus & $\begin{array}{c}\text { MRSA } \\
\text { No. positive(carriage } \\
\text { rate \%) }\end{array}$ \\
\hline Doctors & 46 & $07(15.2 \%)$ & $03(6.5 \%)$ \\
Staff Nurses & 65 & $14(21.5 \%)$ & $04(6.1 \%)$ \\
House Keeping & 39 & $03(7.6 \%)$ & $02(5.1 \%)$ \\
Total & 150 & $24(16 \%)$ & $09(6 \%)$ \\
\hline
\end{tabular}

Table 3: Distribution of S.aureus \& MRSA among healthcare workers of different wards

\begin{tabular}{cccc}
\hline Wards & $\begin{array}{c}\text { Total } \\
\text { no=150 }\end{array}$ & $\begin{array}{c}\text { MRSA } \\
\text { No=9 }\end{array}$ & $\begin{array}{c}\text { S.aureus } \\
\text { No=33 }\end{array}$ \\
\hline ICU & 26 & $2(7.6 \%)$ & $9(34.61 \%)$ \\
NICU & 14 & $1(7.1 \%)$ & $3(21.4 \%)$ \\
Operation Theatre & 61 & $2(3.27 \%)$ & $13(21.3 \%)$ \\
Post-operative ward & 37 & $2(5.4 \%)$ & $5(13.51 \%)$ \\
Emergency department & 13 & $2(15.3 \%)$ & $3(23 \%)$ \\
\hline
\end{tabular}

\section{Table 4: Sensitivity/Resistant Profile of Isolate}

\begin{tabular}{cccc}
\hline Antibiotic Agent & Sensitive & Resistant & $\begin{array}{c}\text { Percentage of sensitive } \\
\text { \% (total No=33) }\end{array}$ \\
\hline Erythromycin & 18 & 15 & 54.5 \\
Clindamycin & 18 & 15 & 54.5 \\
Amoxy-Clav acid & 00 & 33 & 0 \\
Tetracycline & 29 & 04 & 87.3 \\
Moxifloxacin & 23 & 10 & 69.6 \\
Ciprofloxacin & 22 & 11 & 66.6 \\
Vancomycin & 33 & 00 & 100 \\
Linezolid & 33 & 00 & 100 \\
Chloramphenicol & 30 & 03 & 90.9 \\
Trimethoprim-Sulfa & 22 & 11 & 66.6 \\
Cefoxitin & 24 & 09 & 72.7 \\
Gentamicin & 20 & 13 & 60.6 \\
\hline
\end{tabular}

MRSA isolates were sensitive to Linezolid (100\%), vancomycin (100\%) and Tetracycline (75\%).

\section{Risk factors associated for MRSA colonization}

None of the risk factors assessed were significantly associated with the carriage rate of MRSA. Highest positive rate was observed in the age group 31 to 40 years (8.3\%), followed by 21 to 30 years $(6.8 \%)$ and 41 to 50years (2.6\%) ( $\mathrm{p}>0.05)$. The MRSA prevalence rate for male HCWs 6.8 
Table 5: Risk factors associated with MRSA colonization during the study period

\begin{tabular}{cc}
\hline Associated factor & MRSA carriers No(\%) \\
\hline Age group & $5(6.8 \%)$ \\
$21-30$ & $3(8.3 \%)$ \\
$31-40$ & $1(2.6 \%)$ \\
$41-50$ & $0(0 \%)$ \\
$51-60$ & \\
Sex & $5(6.8 \%)$ \\
Female & $4(5.4 \%)$ \\
Male & \\
Ward/Deparment & $2((7.6 \%))$ \\
ICU & $1(7.1 \%)$ \\
NICU & $2(3.27)$ \\
Operation theater & $2(5.4 \%)$ \\
Post-operative ward & $2(15.3 \%)$ \\
Emergency department & \\
Profession/Occupation & $3(6.5 \%)$ \\
Doctors & $4(6.1 \%)$ \\
Nurses & $2(5.1 \%)$ \\
housekeeping &
\end{tabular}

$\%(4 / 58)$ and female HCWs $5.4 \%$ ( 5/92) which was not statistically different $(\mathrm{p}>0.05)$ (Table 5).

Discussion: It is necessary to detect the MRSA carriers among the apparently healthy hospital personnel, particularly those working in the critical care areas. These individuals act as a potential source of infection to their patients, resulting in their extended stay in the hospital. This can be controlled by the regular screening of the HCWs and treating them till they become negative for MRSA.

The carriage rate of MRSA was $6 \%$ in the present study. This was similar with the internationally reported range of the MRSA carriage (5.8 to 17.8\%) among the HCWs in the hospital setting by Shakya B et al. ${ }^{12}$ Nasal carriage rate of MRSA higher (12.7\%) in Aqumas shibabaw et al, ${ }^{9}$ (11.43\%), Sharon et al ${ }^{13}$ and lower (2.32\%) in Shrestha B et al ${ }^{14}$ than this study. These differences can be attributed to variations in microbiological methods (sampling technique, culture and method of MRSA identification), local infection control standards and the local prevalence of MRSA.

In our study, we found that $22 \%(33 / 150)$ of the HCWs carried S.aureus in their anterior nares which is similar with the study reported by Sharon rainy $e^{~} \mathrm{al}^{13}$. S.aureus carriage rate is higher (44.1\%) in Agumas shibabaw et al ${ }^{9}$ study than in our study.

S. aureus carriage rate was highest among Nurses $(21.5 \%)$ whereas MRSA carriage rate was highest among Doctors (6.5\%) followed by Nurses (6.1\%) in this study. Similar results have been reported by Shibabaw et $a l^{9}$ and Malini J et al ${ }^{1}$. High risk of colonization with MRSA strains among doctors and nurses may be due to their frequent patient contact. The susceptibility testing of MRSA isolates revealed high resistance towards gentamycin and erythromycin (42.9 \% each). Low resistance towards ciprofloxacin (14.3\%) and cotrimoxazole (28.6\%) indicates these antibiotics might be an option for empirical therapy of MRSA infections at our hospital.
Obviously, all the above mentioned studies are not fully comparable, since the use of HCWs from specific specialties, and the differences in the study design such as sample size and method of MRSA identification might account for the disparity in the carriage rate. In additions, carrier rates might be influenced to poor personal hygiene of study participants, poor sanitation of the hospital and difference in sampling techniques.

\section{Limitation of study}

Recent diagnostic methods for staphylococcus identification are not available in our institute.

\section{CONCLUSION}

In hospitals, HCWs nasal carriage of MRSA must be regularly screened and should be given warning of the presence of antimicrobial resistant pathogens. Measures to be taken to control the spread of MRSA infection which include: laboratory based surveillance, isolation of colonized and infected patients, use of barrier precautions and basic infection control measures and screening /treatment of MRSA-positive HCWs.

\section{REFERENCES}

1. Malini J, Shruti A. Harle, Padmavathy M., Umapathy B.L., Navaneeth B.V. Keerthi Mannan J., Girish M.S. et al., Methicillin-resistant Staphylococcus aureus carriage amongst Health Care Workers Journal of Clinical and Diagnostic Research. 2012;6(5):791-3

2. Aires-de-sousa $M$, Conceicao $T$, de Lencastre $H$ : Unusually high prevalenceof nosocomial panton valentine leckociden-positive Staphylococcusaureus isolates in CapeVerde islands. J Clin Microbiol. 2006,44(10):3790-3. http://dx.doi. org/10.1128/JCM.01192-06 ; PMid:17021112 PMCid:PMC1594790.

3. Ahmed MO, Elramalli AK, Amri SG, Abuzweda AR, Abouzeed YM: Isolationand screening of methicillin-resistant Staphylococcus aureus from healthcare workers in Libyan hospitals. East Mediterr Health J. 2012,18(1):37-42 PMid:22360009.

4. Omuse G, Kariuki S, Revathi G: Unexpected absence of meticillin-resistant Staphylococcus aureus nasal carriage by healthcare workers in a tertiaryhospital in Kenya. J Hosp Infect. 2012,80(1):71-3. http://dx.doi.org/10.1016/j. jhin.2011.09.009; PMid:22104475

5. Truong $H$, Shah SS, Ludmir J, Twananana EO, Bafana M, Wood SM, Moffat $H$, Steenhoff AP: Staphylococcus aureus skin and soft tissue infections at a tertiary hospital in Botswana. S Afr Med J. 2011,01 (6):413-6.

6. Kumar P, Shukla I, Varshney S: Nasal screening of healthcare workers for nasal carriage of coagulase positive MRSA and prevalence of nasal colonization with Staphylococcus aureu.Biology and Medicine 2011,3(2):182-6.

7. Gupta N, Prakash SK, kumar V," Community acquired Methicillin Resistant Staphylococcus aureus:a new threat for hospital outbreak",Indian journal of patho.microbology. 1999, 42(4):421-6

8. Kirecci E, Miraloglu M: A research of nasal methicillin resistant/ sensitive Staphylococcus aureus and pharyngeal beta-hemolytic streptococcus carriage in Midwifery students in kahramanaras. Turkey Ethiop J Health Dev. 2010,24(1):57-60.

9. Shibabaw A, Abebe T, Mihret A. Nasal carriage rate of methicillin resistant Staphylococcus aureus among Dessie Referral Hospital Health Care Workers; Dessie, North east Ethiopia. Antimicrobial Resistance and Infection Control 2013, 2:25 http://www.aricjournal.com/content/2/1/25 : http://dx.doi.org/10.1186/20472994-2-25 ; PMid:24088259 PMCid:PMC3851550.

10. Kateete DP, Kimani CN, Najjuka FC,"Identification of Staphylococcus aureus: D Nase and Mannitol salt agar improve the efficacy of the tube coagulase test,"Annals of clinical Microbiology and Antimicrobials 2010,9(1);23 http:// dx.doi.org/10.1186/1476-0711-9-23 ; PMid:20707914 PMCid:PMC2927478.

11. Clinical and Laboratory Standards Institute (CLSI). Performance standards for Antimicrobial susceptibility testing. Twenty-third informational supplement. M100-S23, January 2013;

12. Shakya B, Shrestha S, Mitra T. Nasal carriage rate of methicillin resistant Staphylococcus aureus at the National Medical College Teaching Hospital, Birgunj, Nepal. Nepal Med Coll J 2010;12(1):26-9. PMid:20677605.

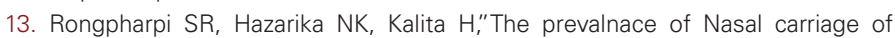
Staphylococcus aureus among health care workers at a tertiary care hospital in Assam with special reference to MRSA" Journal of Clinical and Diagnostic Research. 2013,7(2):257-60 PMid:23543837 PMCid:PMC3592287.

14. Shrestha B, Pokhrel BM, Mohapatra TM. Staphylococcus aureus nasal carriage among health care workers in a Nepal hospital. Brazilian J Infect Dis. 2009; 13(5):322. http://dx.doi.org/10.1590/S1413-86702009000500001; PMid:20428628. 
Rutvi et al: Nasal carrier rate of methicillin resistant staphylococcus aureus(MRSA) among health care workers

Cite this article : Rutvi V, Sangeeta PD, Sima BK, Piyush PA. Nasal Carriage Rate of Methicillin Resistant Staphylococcus aureus (MRSA) among Civil Hospital Health care workers. Int J Med. Public Health. 2016;6(4):180-3. 